\title{
CMMAD Usability Case Study in Support of Countermine and Hazard Sensing
}

\section{SPIE Defense Security \& Sensing}

\author{
Victor G. Walker \\ David I. Gertman
}

The INL is a

U.S. Department of Energy

National Laboratory

operated by

Battelle Energy Alliance

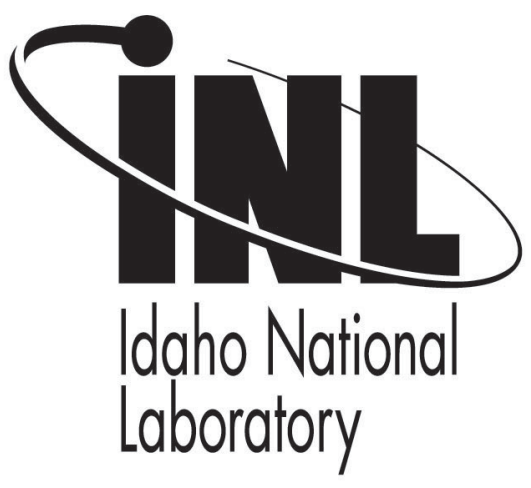

This is a preprint of a paper intended for publication in a journal or proceedings. Since changes may be made before publication, this preprint should not be cited or reproduced without permission of the author. This document was prepared as an account of work sponsored by an agency of the United States Government. Neither the United States Government nor any agency thereof, or any of their employees, makes any warranty, expressed or implied, or assumes any legal liability or responsibility for any third party's use, or the results of such use, of any information, apparatus, product or process disclosed in this report, or represents that its use by such third party would not infringe privately owned rights. The views expressed in this paper are not necessarily those of the United States Government or the sponsoring agency. 


\title{
CMMAD Usability Case Study in Support of Countermine and Hazard Sensing
}

\author{
Victor G. Walker*a and David I. Gertman ${ }^{\mathrm{a}}$ \\ ${ }^{a}$ Idaho National Lab, P.O. Box1625, Idaho Falls, ID 83415
}

\begin{abstract}
During field trials, operator usability data were collected in support of lane clearing missions and hazard sensing for two robot platforms with Robot Intelligence Kernel (RIK) software and sensor scanning payloads onboard. The tests featured autonomous and shared robot autonomy levels where tasking of the robot used a graphical interface featuring mine location and sensor readings. The goal of this work was to provide insights that could be used to further technology development. The efficacy of countermine systems in terms of mobility, search, path planning, detection, and localization were assessed. Findings from objective and subjective operator interaction measures are reviewed along with commentary from soldiers having taken part in the study who strongly endorse the system.
\end{abstract}

Keywords: Human-Robotic Interaction, Interface Design, Countermine, Robotics

\section{INTRODUCTION}

The CMMAD (Countermine, Mobility, Marking, Autonomy and Detection) effort is an INL led effort which is conducted under the auspices of the Project Manager for Countermine and Explosive Ordinance Disposal (PM-CM \& EOD) Autonomous Mine Detection System (AMDS) Risk Reduction Program. The AMDS program seeks to produce an autonomous mine detection vehicle that achieves the same performance of a handheld mine detector. The primary focus of the AMDS program is on the development of novel sensors and data processing algorithms capable of detecting mines which in future phases of this program can be integrated on existing and future robotic platforms. A secondary focus is the reduction of risk associated with the integration of such sensors on robotic vehicles. The latter was addressed through the CMMAD effort, which had the objective to reduce risk for future AMDS phases by assessing the feasibility of autonomous countermine systems through the evaluation of five Critical Interest Functions (CIF). These Critical Interest Functions, which were identified by the government as being essential for robotic autonomous mine detection, are:

1. Plug-n-Play: to what extent can control systems, sensors and manipulators easily be swapped between different robotic platforms?

2. Autonomy: to what extent can autonomous countermine systems successfully locate and avoid mines?

3. Situational Awareness: to what extent can operators of autonomous countermine systems successfully deploy and interact with such systems?

4. Platform Mobility: to what extent can robotic countermine systems successfully traverse a range of terrains?

5. Marking: to what extent can robotic countermine systems accurately and reliably mark mines and safe paths?

The remainder of this paper will describe efforts and investigations associated with the Situational Awareness CIF and how it was assessed in evaluations at a US Army test site. Further details concerning the CMMAD effort and the system design and performance are available in the companion SPIE papers ${ }^{1,2}$.

The situational awareness case studies performed as part of the CMMAD effort inform the AMDS program regarding the feasibility of a modular robot control payload that supports multiple operational requirements from a system usability and cognitive workload perspective. Evaluations, data collection and lessons learned under the CMMAD Situational Awareness CIF will influence next generation robotic countermine and hazard detection system designs to yield systems that are more task-effective and require less cognitive workload to operate. 


\subsection{System Overview}

The CMMAD effort required the creation of system instances to test and evaluate relevant CIF functions and provide a platform for developing key system behaviors and interaction methodologies. These systems were designed and implemented by a team at the Idaho National Lab in conjunction with industry and academic partners.

The systems utilized in the evaluations described in this paper included an iRobot Packbot and a Qinetic Talon both mounted with an electronic computational payload (eBox), and 2-degree of freedom sensor scanning payload (SSP) arm. Both of these additions were created in conjunction with Carnegie-Mellon University and Edge Robotics ${ }^{2}$. Both the eBox and SSP were electro-mechanically designed to be portable across robotic platforms. The systems utilized an 802.11a Bullet Radio for long range Ethernet communications and Bluetooth technology for local line-of-sight communications. The Packbot offered a remote video feed whereas the Talon was instrumented with a spray marking system for physically marking detected hazards.

The overall system supported various sensors depending on mission criteria. For the experiments discussed in this paper a Cytera AN/PSS-14 land-mine detection system was used for mine detection and metal cache localization, the ICX FIDO sensor was used for explosive detection, and the RAE Systems MulitRAE sensor was used for chemical detection.

\section{DESIGN PRINCIPLES}

\subsection{Interface Design}

Through several years the Idaho National Lab has developed software to make robots more useful in real-world situations and strategic missions. The robotic software developed at the INL has been referred to as the Robotics Intelligence Kernel (RIK). The RIK software provides a foundation for executing intelligent behaviors across a widevariety of commercial robotic platforms. The RIK provides a layered approach which allows intelligent behaviors to build on top of generic robot definitions which build on specific platform interfaces. This allows behaviors and control methodologies to be used across several robot platforms with minimal affect on the user experience.

The intelligence built into the RIK software also allows for dynamic changes in how a user interacts with the robot. This is often referred to as dynamic or seamless autonomy ${ }^{3}$. A user can seamlessly change how much control they take in commanding the system versus how much autonomy is given to the robot to make intelligent control behaviors on its own. For instance, in controlling the robot the user can take complete command in tele-operaration, they can have the robot prevent collisions using laser distance data in safe-mode, they can set a target for the robot to self-direct to in target mode, and in autonomous mode they can set a task goal and let the robot complete all related task behaviors (such as sweeping for landmines over a specific area).

A key element of the INL software approach is improved situational awareness and command modules. The RIK system provides several key advantages in both aspects. The system provides interface layers which send key information to interactive systems such as an operator control unit (OCU). In addition, the RIK allows command interfaces from several types of tools to control the robot. The following section describes a few of these situational awareness and tasking interfaces provided on the robots tested in this exercise. Several other control and system feedback technologies have been developed with the RIK but were not utilized in these field observations.

\subsection{Situational Awareness}

Situational Awareness deals with the ability of the user to understand the environment in which the robot is operating. This includes the relevant constraints of the robot, the significant sensor readings associated with the current mission, location of the robot in the physical world, and feedback from commands sent to the robot. Situational awareness also includes the operator's perception of environmental conditions, comprehension of their meaning, and ability to project conditions and status to the near future ${ }^{4}$. Thus, situational awareness is a dynamic, updatable process.

In designing interface and situational awareness feedback systems, the INL team has focused on providing relevant information so a user can quickly make a system decision and understand the most important elements of the robotic environment. The total efficiency of a system will likely always include a user for providing task definition and control as well as receiving mission feedback. 
Some relevant situational feedback mechanisms utilized in the evaluations included the following:

\section{Video:}

Video provides visual clues to the environment that the robot is operating in and the movement of the robot and the arm in that environment. In the hazard mission the robot had a wireless transmitting camera mounted to the top of the arm that provided visual feedback on the location of the robot and arm. It was a fixed camera and did not pan with the arm. The countermine mission did not include a video feed.

\section{Sensor Readings}

Readings from various sensors on the robot provide direct feedback in the form of numeric values. These values are provided with an adjustable color-coded scale to indicate the strength of the reading, and an indicator of the lowest and highest values encountered. The users can choose which sensor readings to display

\section{Occupancy Map}

This provides a dynamic map of physical elements in an environment. Each of the robots was equipped with LIDAR laser sensors to detect the presence of objects in the environment and their distances. This information is translated into an occupancy map of physical objects which can represent the world from the robot's perspective and the robot's location in that map.

\section{Sensor Map}

In addition to sensor reading values, the corresponding color-coded scale for different values can be painted onto the physical occupancy map. This provides lasting clues to patterns in sensor readings and their correlation to the physical environment and enhances overall situation awareness of sensor data which is usually not visible. See Figure 1 for an example of the interface with a sensor map.

\section{Line of Sight}

In some missions there is opportunity for the operator to be within visual range of the robot and its operating environment. In other environments visibility is obscured and the user is completely dependent on information sent from the robot itself. Both these conditions were tested in the case studies to learn of the impact to situational awareness for each of the interfaces.

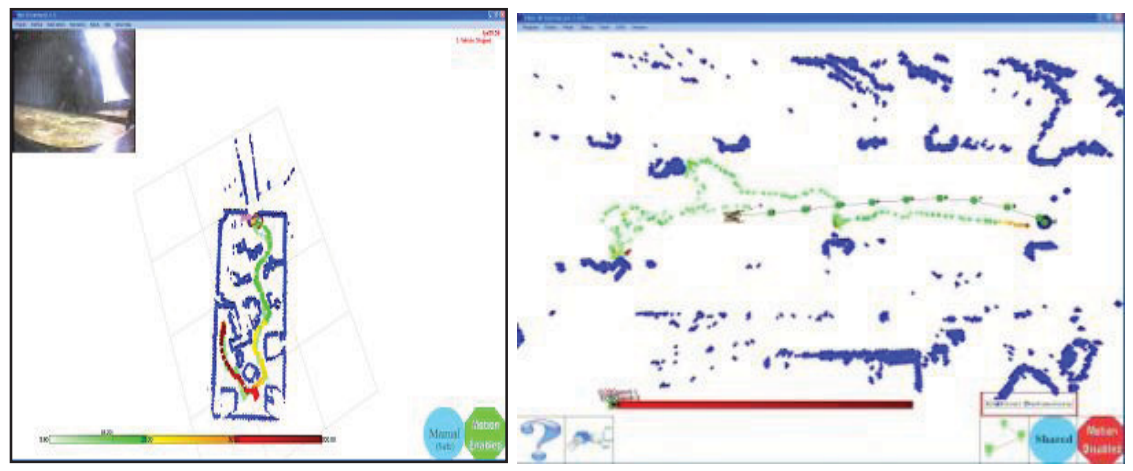

Figure 1. Physical map and sensor readings on operator OCU. a) indoors and b) in the field environment

\subsection{Control Interfaces}

The RIK system supports several types of command methods and tools to control the robot. Interfaces were designed to support easy and natural interactions with the robot and associated tools ${ }^{3}$. Most of the interfaces can be customized, and use different types of technologies and methods. For these case studies, the following command interfaces and approaches were tested: 


\section{Laptop / X-box Controller (Safe Mode / Tele-operation)}

This command interface consisted of a laptop with a proprietary interface which included a physical and sensor map and video (where applicable). Users could use the keyboard or an x-box controller to command the robot. The left control stick on the controller moved the robot and the right control stick moved the SSP arm. The robot included intelligent behaviors which prevented collisions, such as regulating speed as the robot approached physical objects and not allowing the robot to move into potentially occupied space with the chassis or arm. Users could chose to change the dynamic autonomy mode to "teleoperation" and disengage robot's safe-motion behaviors to take full control of the robot and SSP arm motion.

This safe-teleoperation with x-box controller and maps was often referred to as "Shared Mode" by the subjects.

\section{Laptop / Target Mode (Tasking / Autonomous)}

This command interface also used the laptop which included a map and video. In this mode, users would utilize a mouse to drop a "target" location on the map for the robot to move to. The robot intelligently plotted the path to the target and drove there without need for further input from the user. It would avoid obstacles and continue to map surrounding objects and collect sensor readings while navigating to the target. After reaching a desired location the users could switch to the x-box controller or keyboard to control the SSP arm and finely position the robot for detailed investigations.

\section{Wii Controller / Video \& Sensor feedback (Safe Mode / Tele-operation)}

This interface focused on using a wii-mote controller which connected directly to the robot via Bluetooth technology without the need for a base station, radio or laptop. The wii-mote and nunchuck attachment were mounted into a wii-rifle for ease of holding, and a small tablet PC was mounted onto the front of the rifle for viewing of video and sensor readings. The robot movement was controlled with the wii-mote's buttons and the SSP arm was controlled with the nunchuck attachment. This method did not provide map feedback; it focused only on video and sensor readings.

\section{FIELD STUDY DESIGN}

\subsection{Overview}

The studies took place over a two week period at a US Army test site. The observation courses were located on the explosives test ranges. The primary range for the first 3 scenarios was along a simulated concrete roadway which had occasional mines and metal clutter beneath the surface. The course simulated what might be found in an urban area with potholes, loose perimeters, railings, and elements such as boxes, bags, tires, and hay bales to serve as obstacles and targets for investigation. A $12^{\prime}$ by 20 ' shelter was used to provide subjects protection from weather conditions and to accommodate; line-of-sight (LOS) and non-line-of-sight (NLOS) conditions. A secondary simulated environment involved a concrete culvert with collections of objects inside. And finally, a field with inert land mines buried in patterns was used for the countermine demonstration.

\subsection{Mission Scenarios}

The mission scenarios were designed to leverage advantages of the test area, sensor, robot and tasking technology and availability of subjects.

For scenarios 1-3 metal shells or explosives (TNT/C4) were hidden in targets and to be detected using an AN/PSS-14 or FIDO sensor scanning payload on the SSP arm. During the testing, the subjects controlled the robot through one of three tasking modes: target mode using the OCU/Map and mouse, safe-teloperation mode using the OCU/Map and X-box controller (often referred to as shared mode), and safe-teleoperation using the video/sensor viewer and the Wii rifle. The subjects performed the scenarios using either line-of-sight visibility of the course or non-line-of-site with the course deliberately obscured by a tarp covering the control shelter. Scenario 4 used only the Wii rifle control method, and Scenario 5 used only the OCU methods (target mode and safe/shared mode). 
With the exception of scenario 4, subjects and experimenters were positioned in the shelter and collected video of the trials and statistical information about computer use and times for each scenario. Prior to each test, the experimenters ensured that the subjects were familiar with the mission objectives, tasking mode, control of the robot, and information presented to them on the OCU. For all conditions, subjects were encouraged to provide feedback to the experimenters during and after the scenario. Normal time for each condition was 10-15 minutes per session.

\section{Scenario 1: Obstacle course with metal hazard - using ANP/SS-14}

The sparse obstacle course was approximately $35 \mathrm{~m}$ x $10 \mathrm{~m}$ and had hay bales placed intermittently as a perimeter with a few additional hay bales that functioned as obstacles placed strategically throughout the course. This scenario used metallic materials placed in target locations equal distances from a start location. The targets included a pile of rock rubble, a pile of bags filled with hay, and a collection of boxes. The overview of the course and a target are shown in Figure 2.

The soldiers were challenged to navigate the robot to a specific target employing the assigned tasking and visibility mode and inspect it for a suspected metal hazard.

\section{Scenario 2: Obstacle course with chemical hazard - using FIDO}

The obstacle course for this was similar to the one in Scenario 1, but soldiers used the robot equipped with the FIDO chemical sensor to detect explosives. For this scenario, canisters filled with TNT were placed in the assigned targets.

\section{Scenario 3: Search with chemical hazard - using FIDO}

After all the subjects had completed scenarios 1 and 2, they participated in a more open, search-based scenario that built upon their experience over the prior three days. The subjects were asked to investigate 3 targets strategically positioned on the concrete lane and determine if there were explosive chemicals hidden in the targets. Not all of the targets contained hazardous material and the subjects had to determine and report their findings. The course was mirrored, allowing for two different scenarios. Side A included boxes, gunny sacks and a length of guard rail for investigation, side B had a hole in the road covered with straw, tires, and a guard rail. The distances between inspection areas were equivalent and subjects were randomly assigned first to course A or B. Only the OCU-based tasking conditions were used, allowing subjects to utilize target and safeteleoperation with the $\mathrm{x}$-box controller. Since the subjects had had considerable practice with the tasking modes, they were told to use the modes in the manner they saw fit, including switching between modes to accomplish their mission. At the end of each mission subjects returned to home base and reported on their experience via a survey debriefing form and an interview with an experimenter in the test trailer. The subjects ran a trial in both line of sight and non line of sight conditions (one on each side).

\section{Scenario 4: Culvert investigation - using Fido.}

This scenario was primarily a feasibility investigation. It allowed soldiers to use the Packbot and the Wii-rifle controller to examine a concrete culvert which contained boxes and $\mathrm{C} 4$ explosive. The culvert was approximately 3 feet in diameter and was located near the concrete lanes described in scenarios 1-3. See Figure 2. This scenario was a "traveling" mission where the primary control station was left behind and the soldiers followed the robot at a distance during the exercise. They utilized the Blootooth connection with the Wii-Rifle for control and the mini-computer with video and sensor readings for feedback. The goal was primarily to gain insight into the use of the system in a mobile mission and issues concerning controlling the robot into a constrained space.

\section{Scenario 5: Landmine detection - using AN/PSS-14}

This scenario involved the use of the Talon equipped with the AN/PSS-14 and a marking system for land-mine detection. The course was set at a specialized field which included varying terrain, hay bales for a perimeter, and a few barrels to serve as obstacles. This exercise was limited to 3 participants who controlled the system using the OCU methods and target mode or safe-teleoperation mode using the $\mathrm{x}$-box controller. The case studies varied over a simulated "dense" mine-field (a mine every 2.5 meters) and a "sparse" mine-field (a mine every 3.0 meters) as well as line-of-sight and non-line-of-sight. Figure 2 includes a picture of the course. 

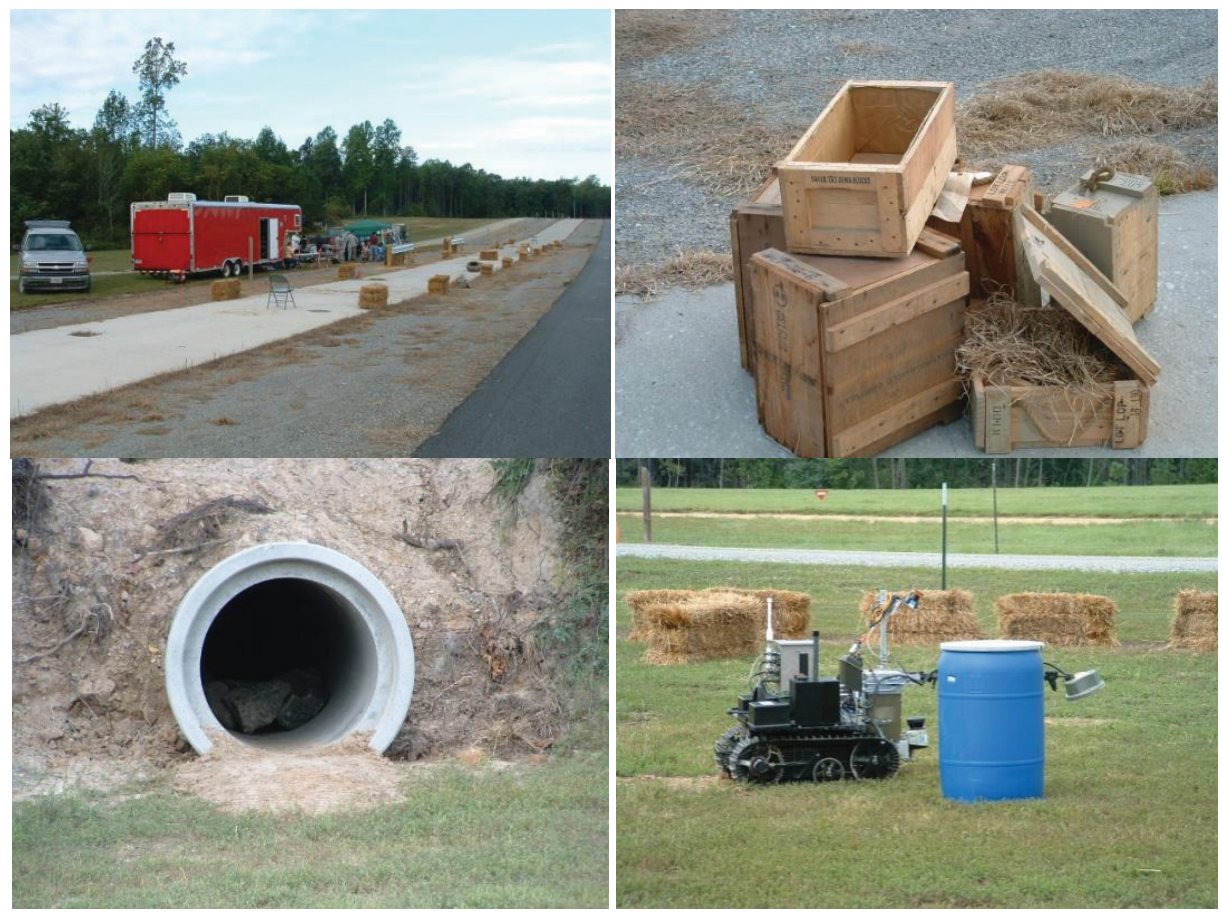

Figure 2. Various course layouts from scenarios 1-5

\subsection{Subjects}

Five soldiers and one UXO Tech III expert served as subjects supporting the evaluation. The ages of the subjects ranged between 23 and 39 years of age. Military service was between 5 years, 6 months to 12 years, 8 months. Four subjects had at least 12 months combat experience in Iraq. Participants' formal computer class room training ranged from between 0 months to one year. All subjects had experience in playing video games. Trials occurred over multiple days and assignment of subjects and ordering of trials was fully randomized.

Note that scenarios 1 and 2 consisted of 6 subjects, scenario 3 had 5 subjects, and scenarios 4 and 5 had only 3 subjects each.

\subsection{Training}

The subjects were shown the robot system in a group setting. They were introduced to the topics, told how the robot was operated and were shown its control and feedback. Subjects were also given simple written materials explaining the operational aspects of the mission they were about to perform and characteristics of the system to be tested. Prior to testing, each subject had the opportunity to drive the robot in all of the described modes of operation. Operators were allowed to ask the research team and experimenters any questions regarding the robot, its operation, and/or capabilities. The questions and answers were not formal, but often evolved into a general discussion. Overall training was less than 45 minutes for each subject.

\subsection{Feedback}

During the training session, operators asked questions, shared thoughts and provided general feedback. Most of the operator comments and responses were recorded on survey forms and are presented later in this paper under findings and discussions. The surveys for each session consisted of 35 questions regarding their experience with the particular tasking mode and operating conditions. The surveys made use of Likert rating scales and some open comments. After completing all sessions for each scenario they were asked a set of debrief questions regarding the overall system attributes for that scenario.

In addition, we recorded a set of metrics from the interface (when available) to understand how the robot and interface was used. This included total mission time, information regarding joystick usage during operation modes, frequency and location of target drops, and switches between tasking modes. 


\section{OBSERVATIONS / FINDINGS}

\subsection{Overview}

Previous research has indicated the importance of observational data associated with usability analysis to further technical development and system design with respect to human robot interaction ${ }^{5,6}$. Thus, both empirical data and observations are presented in these case studies.

Much of the data has yet to be fully analyzed, but in investigating surveys, recorded data, and discussions, several trends were observed in the use and efficiency of the system. Many of the most valuable observations were in subject comments and choices. Overall, the subjects were encouraged with the usefulness of the system and found it effective and easy to apply in the pursuit of mission objectives. Overall, acceptance ratings were positive for the system regardless of the mode or conditions.

Some observations and data findings follow.

\subsection{Workload measures and findings}

Overall workload measures for explosives detection are presented in Figure 3a-b below.

Workload measurements employed the NASA TLX inventory ${ }^{7}$ previously used in HRI usability studies. The survey consisted of a ten point scale where subjects indicated the workload that they experienced after each individual condition. The average working load ratings across 6 workload dimensions presented in the figure below were quite low indicating that the combination of the interface and tasking mode strongly supported mission completion for both FIDO and ANP/SS-14. The subjects' rating of their own performance was slightly above midpoint which is reasonable considering the limited amount of time and experience that they had with the system. Mental and physical workload was low for both metal and FIDO conditions. Summed over all subject ratings, the use of the metal detector imposed slightly more workload than did use of the FIDO, but this trend is not significant. The Subjects indicated a somewhat lower performance rating when in the metal condition that is consistent with slightly greater workload. This effect could be due to training since subjects experienced hazards detection with METAL first and then FIDO on the second day.
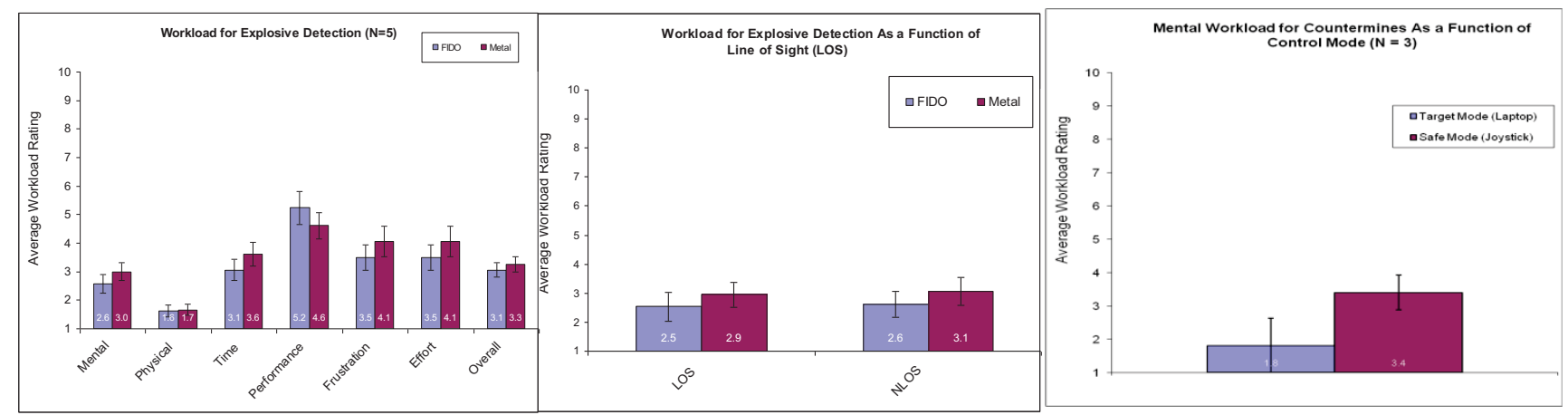

Figure 3. Workload ratings for Scenarios 1-2: a) averaged b) as a function of LOS and c)Scenario 5 by Control Mode

Average workload was further evaluated for line of sight versus non line of sight conditions. Figure 3 above also presents the average workload findings as a function of line of sight for both Metal and FIDO sensor scanning payloads. The data have been collapsed across the three tasking modes, safe-teleoperation, target, and Wii rifle. In general, the low workload associated with the combinations of sensor and line of sight conditions correlate well with the relatively high situation awareness ratings given by subjects. In fact, the interface was good enough that even when line of sight was denied to them, subjects found the workload in the hazards detection task to be quite low.

It should also be noted that there were no statistically significant differences in workload associated with the different tasking methods for scenarios 1 and 2. This may be due to the fact that the overall system presented a strong reduction in workload when compared to similar tasking as conducted in the field. There were definite preferences for tasking modes 
as indicated in subjects' comments in section 4.10, but using one tasking method versus another did not translate into significant changes in workload and situation awareness measures and findings.

Figure 3c, however, shows that tasking method represented a significant difference in mental workload for scenario 5 . In this scenario the mental workload reported by the subjects for target mode was almost half that reported for the safeteleoperation mode. In both modes the system would complete scanning operations, and autonomously classify the mines. It would also produce a virtual wall around the mine which would keep the robot from crossing it while it was in a safety mode. With the shared mode the users were required to determine direction around the mine and wait for completion of each sweeping task before issuing the next command. Users expressed they had to be much more involved in the system during safe-teleoperation so they could determine when the robot had completed autonomous tasks and was ready for further input. In target mode they indicated they need only wait and observe the continued actions of the robot unless it required intervention.

\subsection{Situational awareness measures and findings}

Overall, the operators reported that the system was easy to use and that workload was quite low. This generally indicates that the subject's internal mental model closely matches the functional characteristics of the system and its operation. It also suggests that their situational assessment of real-world conditions is accurate. When there is a mismatch between an operator's mental model and the current situational model, the result is that overall situation assessment is reduced and cognitive workload increased ${ }^{8}$. In order to test how well feedback available supported operator situation awareness, a number of questions were posed to the operators including whether it was easy to recognize objects, easy to recognize robot location, whether the display supported understanding, and whether system status was obvious. Operators indicated their ratings on a 5 point Likert scale with 5 being the most positive. For analysis purposes, it was assumed that a neutral response representing the scale midpoint could serve as a test case against the ratings that were obtained. Findings for these items were determined to be significantly different than chance. Other situation awareness questions pertaining to comprehension were determined to be non- significant.

Figure 4 below represents the average responses for situational awareness and indicates a very high agreement that the feedback provided made it easy for the operator to understand the conditions of the robot in the real-world environment. Note that this was true across all scenarios described above even though the missions were often different in nature.

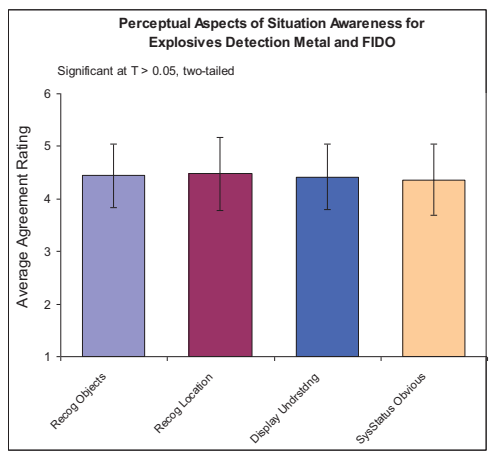

Figure 4. Perception Ratings on a scale of 1-5

\subsection{Task-Based Operating Mode}

Data collected in surveys, comments and observations indicated that the users had preferences in tasking modes which were dependant on the type task they were attempting to complete. Although each tasking mode had areas for which they were well utilized, the preferences differed for the different scenarios, as indicated in figure 5 below

For instance, in scenario 3 the users were encouraged to utilize whatever tasking mode they felt would best support the mission they were performing. The surveys and discussions with the soldiers indicated that when they were in a forward searching mode and felt highly mentally engaged in the task that the robot needed to perform, the majority preferred safe-teleoperation using the $\mathrm{x}$-box controller. One subject preferred using target mode to cover long distances, but all subjects used the $\mathrm{x}$-box controller as part of detailed investigation.

In scenario 3, there was an $80 \%$ preference for utilizing the safe-teleoperation during forward searching investigation, even for transferring between target locations. However, in returning to the home state after the investigation was 
complete the preferences were reversed. $80 \%$ utilized a target to return the robot home. Many indicated that they didn't feel like they needed to be mentally involved at this point and were happy that the robot could return on its own. The only subject to not utilize the target mode later indicated that he had forgotten it and wished he had used it.

Similarly, in scenario 5, when investigating landmines, the majority of the subjects preferred the target mode over safeteleoperation. Several reported that they did not feel they needed to be consistently engaged in the task and preferred the system performing autonomously, only needing to intervene as required.

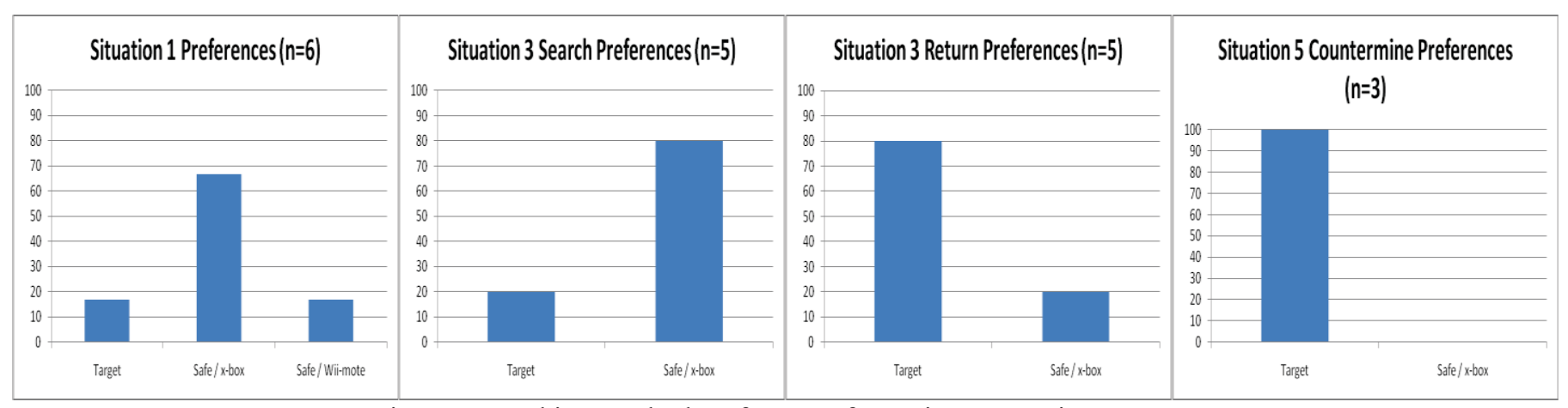

Figure 5. Tasking Method preferences for various scenarios

All tasks which we classified as requiring high-mental resources on the part of users, such as classifying and interpreting readings or determining where to best engage sensors, resulted in users selecting a higher-control method. Their physical engagement seemed to match their mental engagement. If the system could provide classification and intelligent behaviors and provide feedback to the users without mental engagement then the users preferred more autonomous modes such as target mode. However, it was noticed that when subjects had the opportunity to delegate general landmine detection to intelligent behaviors that would classify and mark the mines, the users preferred to simply drop the target and let the system do the rest. There was a trend for users to indicate strong satisfaction with the system when using a command mode that seemed to match their task engagement.

\subsection{Task-Based Joystick Usage}

Data recorded from the system also indicated that the users used the joystick differently depending on the type of task that they were to perform. The Figure 6 below indicates the positions which the subject held the joystick while operating the $\mathrm{x}$-box controller in controlling the robot. The joysticks for driving the robot and moving the arm are both proportional, the further you move the stick from center the faster the motion effected. The SSP arm contains the sensor head used to inspect a suspicious area of interest such as boxes, crates, rock piles, etc. The right figure shows how operators use the joystick incrementally to perform fine arm movements. The left figure shows that operators drive the robot using maximum throw of the joystick in gross movements rather than the fine control movements shown in the arm control figure. The trend was noted across all subjects. The results indicate that that joystick usage is a function of task type, with the same subjects treating the joystick in dramatically different ways depending on the task.
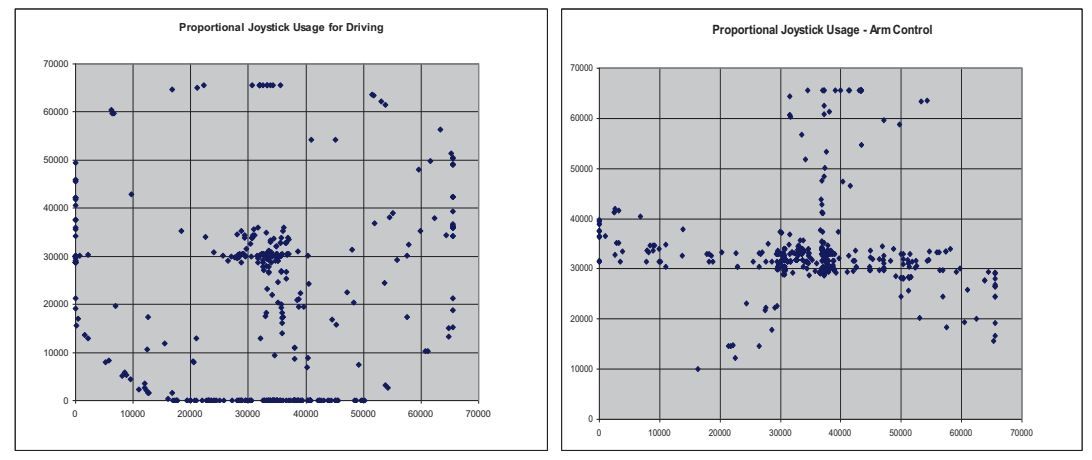

Figure 6. Proportional joystick usage in a) driving the robot and b) controlling the SSP Arm 


\subsection{Should the system be fielded?}

After experiencing the system in different scenarios and mission types each of the users was asked in the debrief surveys if they felt the system was useful in these scenarios and whether the system should be fielded with similar functionality. Figure 7 presents subject ratings strongly endorsing the system for future fielding, all respondents responded in the affirmative.

In comments to the experimenters, one subject asked how long it would take to get the system to the field.

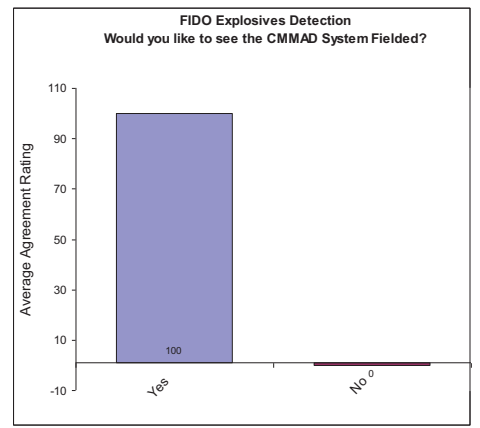

Figure 7. Subject indicating whether the system should be fielded.

\subsection{Scenarios 1-3 observations / limitations: Hazardous material search}

As indicated above, the subjects were very encouraged by the system's utility in the hazard-searching mission. Although they were able to quickly perform the missions, they felt that they improved their operational skills over time. In this mission the AN/PSS-14 was only utilized to provide metal detection readings and ignored the ground penetrating radar readings and landmine classification algorithms. This sensor proved to be an effective indicator which the users were comfortable with. The Fido explosive sensor took more training for users to effectively understand as the readings are more complex and obtaining higher sensor values is very dependent on environmental conditions such as wind and placement of the explosives. Further system intelligence in classification of the readings and indications would likely increase satisfaction with the overall system.

In an outdoor environment the users indicated a greater need for visual clues to aid in situational awareness. They made several suggestions for improved video and more cameras in searching. In addition, the users seemed to find the mapping capabilities very valuable in areas where there were clear features. The mapping capabilities have proven very useful in indoor environments, and in the outdoor conditions they clearly helped the subjects return to locations they had already investigated and to the home position. In scenarios 1-3 there was a slight incline in the course which could provide interference so the mapping would likely have been clearer if the laser had been mounted higher.

Some subjects indicated that they would benefit from more intelligent behaviors associated with the hazard missions, such as more automatically stowing the arm when targets are dropped to allow for easier handling, and more classification indicators.

\subsection{Scenario 4 observations: Capability exploration involving a culvert exploration task}

Subjects were able to use the Wii-rifle and successfully control the robot over an extended travel distance and perform search of the culvert. The robot was able to successfully enter the culvert, sniff the boxes for explosives and return by backing out.

The scenario indicated that the angle of entry in such a confined space was important for allowing the robot to properly position itself. The use of the Wii-rifle proved valuable in the walking phase with subjects indicating the ease of following the robot with the simple controllers. However, the screen was difficult to read in the daylight, making video and sensor readings difficult to see clearly.

\subsection{Scenario 5 observations: Countermine assessments}

The countermine exercise was shortened by system and weather issues, but the subjects who participated gave valuable feedback on the system's operational aspects in a countermine mission. The subjects indicated low workload and high 
efficiency with the system as a whole. They felt that the system did a good job of indicating the presence of a mine and properly moving to avoid it. Some of the behaviors associated with marking were not functioning and there were suggestions to enhance those attributes. They also suggested clearer indications of the robot taking autonomous action (which has since been implemented).

The Talon system tested in this exercise did not have a video feed and the laser was mounted low to the ground so that grass in the field was mapped as physical objects as well, making the map seem cluttered and difficult to interpret. Subjects indicated the need for video and enhancing the physical map customizing to increase situational awareness. Overall they felt the system behaviors were valuable and unanimously agreed that the system should be deployed in the field.

\subsection{Operator debrief comments}

Comments were collected during debriefing and provided valuable insight to the user experience. A sample of these comments includes the following:

Related to trust: "Yes, I trust the system", "I trust the system completely", "I can see myself using it and going into theatre", "I trusted the robot, it's simple to use once it gets running", "Yes I trusted the robot, it did what I told it to with little assistance."

Related to usage : "I used target when I know the object was far away, As I got close, I used the shared mode", "I liked and used the shared mode of tasking more often, I used target to bring things back home", "I used shared mode to navigate", "The more I used the system the better I became at it", "Concepts in driving and directing the robot could be used to interrogate explosive hazards", "Would like to see the system fielded. It could be used in a convoy to send the robot ahead if you think there might be a [hazard]", "After 3 days usage, I became comfortable with system."

Related to enhancements and suggestions: "The motion of the arm speed (horizontal speed) needs to be slowed down, otherwise working on an explosive source you could damage or create a dangerous situation", "It would be nice for the system to issue some sort of a sound confirming explosives detection", "Add battery information on the interface so operators know how much time they have left", "Next stage in development of the system - 'Ruggedize'", "I'd like to be able to pan as I travel around the explosive source to check for additional hazards", "The robot showed blades of grass as obstacles. Having a camera would improve this. I would not run the robot without this" (related to scenario 5).

Trust in the system was unanimous across subjects. Two of the subjects indicated that the testing made them feel that the system would be appropriate in urban scenarios including situations stateside.

\section{CONCLUSION AND DISCUSSION}

Robot technology in support of the war-fighter has an incredible future and advances in robot behaviors, autonomy, display integration and tasking have the potential to support military mission objectives. The field studies performed here indicates that soldiers are able to effectively utilize intelligent robotic systems in realistic scenarios with different types of missions.

Case studies performed in the field allow for soldiers to use their personal experience and knowledge to aid in the design and evaluation process. In this study, they provided valuable input regarding allocation of function between human and machine, evolving useful user dialog, accommodating environmental factors, providing displays and controls consistent with operator tasks, identifying key elements of situation awareness and workload, and enhancement of performance through use of effective and consistent icons, colors and data.

The studies performed indicate that these robot systems are most valuable with several flexible methods of commanding the system and obtaining feedback. Different types of tasks were best suited to different levels of autonomy, control and tools. The RIK software interface provided the necessary ability to integrate these different tools and methodologies into one system.

For the scenarios examined, workload ratings were low across the majority of tasking conditions including line of sight and non line of sight indicating the relative success of the integration of the robot behaviors and the OCU. When using the system, subjects' perceptual ratings of situation awareness for four distinct features; display understanding, system status, recognition of objects, and recognition of location, were very positive and significant. Training was relatively 
brief but effective; suggesting that the system tested was easy to comprehend for first time users. Overall trust in the system was unanimous across the users. Further, the participants suggested that the system should be fielded. Additionally, subjects noted a number of system improvements including arm stowage, battery indication, and use of cameras in conjunction with mapping lasers which could be integrated into future deployments.

In general, the system performed well for users and they were very supportive of adding intelligent behaviors to the robot to ease their burden when performing particular tasks. .

We conclude that there is value in additional studies associated with natural mapping of autonomy levels to task engagement across a wide variety of tasks. Furthermore, suggestions on arm control indicate a need for potential development and associated studies to seamlessly integrate what are now separate systems for manual control of the robot body and arm. This integration effort would require additional, highly controlled experiments and understanding of the finer aspects of motion in separate systems toward a common target.

Continued work in understanding the interactive aspects of intelligent systems and user interaction, including seamless autonomy in support of achieving mission-centric objectives offers us the ability to reduce operator workload and enhance situation awareness, increase the quality of data, and support robotic advancement that would be applicable across an even greater variety of scenarios.

\section{ACKNOWLEDGEMENTS}

Funding for the CMMAD effort was provided through the AMDS program which was administered through US Army RDECOM CERDEC Night Vision and Electronic Sensors Directorate, with support from the Institute for Defense Analysis (IDA) We acknowledge the support and guidance of the program manager (Ed McAuley) as well as that of the Night Vision team (Mark Locke, Eloisa Lara and Matt Aiello) and the IDA team. The support of multiple staff members at INL and in the different institutions and organizations participating in the CMMAD effort is gratefully acknowledged.

\section{REFERENCES}

[1] Few, D., Versteeg, R., Herman, H.. "Semi-autonomous mine detection system," 2010 SPIE Defense Security and Sensing Meeting. Orlando, Florida (2010).

[2] Herman, H., E.T. Henciak, M.J. Licitra, J.D. McMahill, J.V. Valois, D. Few, and R. Versteeg. "Modular Countermine package for small robots," 2010 SPIE Defense Security and Sensing Meeting. Orlando, Florida (2010).

[3] Few, D. A., Bruemmer, D. J., Walkton, M. C.. "Seamless Autonomy: Removing Autonomy Level Stratifications," HSI 2008, Krakow, Poland (May 2008).

[4] Endsley, M. and Garland, D. Situation Awareness, Analysis and Measurement], Lawrence Erlbaum Associates, Publishers, Mahway, New Jersey (2000).

[5] Few, D. A., Smart, W.D., Bruemmer, D. J., Nielsen, C. W. "What Does it do?: Human-Robot Interaction Experiments with the General Public," Proc. of the IEEE International Symposium on Robot and Human Interaction, Jeju Island, South Korea (Sep 2007).

[6] D. A. Few, Bruemmer, D. J., Marble, J. L.. "Usability and Portability Lessons Learned from the 2003 AAAI Robot Rescue Competition," 2003 American Association for Artificial Intelligence Mobile Robot Workshop, Acapulco, Mexico (August 2003).

[7] Hart, S., \& Staveland, L. "Development of NASA-TLX (Task Load Index): Results of theoretical and empirical research," [Human Mental Workload. Advances in Psychology], P. Hancock \& N. Meshkati (Eds.), Amsterdam: North-Holland.ISO 13407, 52, 139-183. (1999).

[8] O’Hara, J. M., Higgins, J. C., Stubler, W. F., Kramer, J. (2000) “Computer_Based Procedure Systems: Technical Basis and Human Factors Guidance, NUREG/CR-6634, Washington DC. 